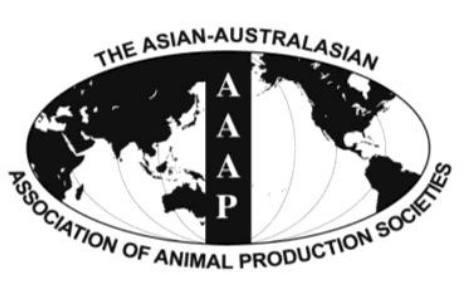

Open Access

Asian Australas. J. Anim. Sci.

Vol. 27, No. $10: 1417-1425$ October 2014

http://dx.doi.org/10.5713/ajas.2014.14206

www.ajas.info

pISSN 1011-2367 elSSN 1976-5517

\title{
Pig Spermatozoa Defect in Acrosome Formation Caused Poor Motion Parameters and Fertilization Failure through Artificial Insemination and In vitro Fertilization
}

\author{
Won Young Lee, Ran Lee, Hee Chan Kim, Kyung Hoon Lee, Xiang Shun Cui ${ }^{1}$, Nam Hyung Kim ${ }^{1}$, \\ Sang Hyun Kim ${ }^{2}$, Il Joo Lee ${ }^{2}$, Sang Jun $\mathrm{Uhm}^{3}$, Min Jung Yoon ${ }^{4}$, and Hyuk Song* \\ Division of Food Bioscience, RIBH, College of Biomedical and Health Sciences, \\ Konkuk University, Chung-ju 380-701, Korea
}

\begin{abstract}
The selection of morphologically normal spermatozoa is critical to obtain high breeding performances in boar breeding farms and artificial insemination (AI) centers. Parameters for the selection of semen mainly include total sperm motility, concentration, and morphology. However, these primary parameters are often not reliable for discriminating between normal and abnormal, nonfertilizable spermatozoa. The present study was designed to compare the motion characteristics, fertilization ability using in vitro fertilization (IVF), and acrosome formation of the semen from boars having low (boar number 2012) and normal (boar number 2004 and 2023) breeding performances. The ultimate goal was to identify additional simple and easy criteria for the selection of normal sperm. There was no significant difference between boar 2004 and boar 2023 sperm total motility in computer assisted sperm analysis. However, boar number 2012 semen presented a significantly reduced population of rapid moving spermatozoa and an increased population of slow moving spermatozoa compared to boar numbers 2004 and 2023. Analysis of detailed motion characteristics revealed that sperm from boar number 2012 had significantly reduced motility in progressiveness, average path velocity, straight-line velocity (VSL), curvilinear velocity (VCL), straightness, and linearity. The assessment of the fertilizing ability by IVF also showed that sperm from boar number 2012 showed a fertility rate of 3.4\%, whereas sperm from boar number 2023 had a fertility rate of $75.45 \%$. Interestingly, most of the sperm nuclei were found on the peripheral area of the oocytes, suggesting that the sperm from boar number 2012 lacked penetration ability into the oocyte zonapellucida. The acrosome formation analysis using Pisum sativum agglutinin staining demonstrated that the sperm from boar number 2012 had a defect in acrosome formation. Consequently, primary parameters for selecting semen before AI such as motility are not sufficient to select normal and fertilizable spermatozoa. In conclusion, the present study suggests that the acrosome staining and detailed motion characteristics such as progressiveness, VCL, and VSL should be included in determining semen quality together with primary parameters for successful AI and high breeding performance in the swine industry. (Key Words: Boar, Sperm, Motion Parameters, In vitro Fertilization, Acrosome Defect)
\end{abstract}

\section{INTRODUCTION}

Artificial insemination (AI) has been widely used in the

\begin{tabular}{l}
\hline * Corresponding Author: Hyuk Song. Tel: $+82-43-840-3522$, Fax: $+82-43-$ \\
$842-3522$, E-mail: lovelyhusband @kku.ac.kr \\
${ }^{1}$ Department of Animal Science, College of Agriculture, Chungbuk \\
National University, Choung-ju 361-763, Korea. \\
${ }^{2}$ R\&D Team, Darby Genetics Inc. Anseong 456-915, Korea. \\
${ }^{3}$ Department of Animal Science and Biotechnology, Sangji Youngseo \\
College, Wonju 220-713, Korea. \\
${ }^{4}$ Department of Horse, Companion and Wild Animal Science, College of \\
Animal Science, Kyungpook National University, Sangju 742-711, Korea. \\
Submitted Mar. 20, 2014; Revised Apr. 24, 2014; Accepted May 27, 2014
\end{tabular} swine industry, and the quality of spermatozoa is the most critical factor for successful AI. The assessment of spermatozoa quality before AI has been performed by assessing a number of basic parameters based on sperm concentration, motility, and morphology (World Health Organization, 1992; Correa and Zavos, 1994), and most of the AI centers in Korea rely on these primary parameters for determination of sperm quality. However, many studies have shown the limitations of these primary parameters in the assessment of sperm quality. Although these parameters have been regarded as criteria of normal spermatogenesis and fertilization potential (Saacke, 1983; Amann, 1989), 
they cannot be used as reliable predictors of fertilizing ability (Glass and Ericsson, 1979; Blasco, 1984; Bostofte et al., 1984; Foldesy et al., 1986; Hoing et al., 1986; Amann, 1989). Fertilization disorders due to poor sperm quality result in huge monetary loss in both $\mathrm{AI}$ centers and swine farms. To avoid this kind of unpredicted economic loss, a functional assessment of sperm quality is necessary. Several methods have been developed such as cervical mucus penetration, oocyte penetration assay, and measurement of acrosin activity (World Health Organization, 1992). However, these methods require labor, and time consuming and specialized equipment that restrict field applications.

The common sperm defect that prevent normal fertilization through AI in large animal is an acrosome defect, the acrosomes are knobbed, ruffled, and incomplete (Chenoweth, 2005). The acrosome defect-mediated infertility has been reported in human (Aitken et al., 1990; Moretti et al., 2005), boars, bulls, stallions, rams, and dogs (Nicander and Bane, 1966; Hurtgen and Johnson, 1982; Toyama and Itoh, 1993; Soderquist, 1998; Chenoweth, 2005; Santos et al., 2006). Identification of defective spermatozoa in early stage before AI is important for maintaining semen quality and good prolificacy.

In this study, the fertilization ability and detailed sperm motion characteristics, besides total motility and viability, from normal and low breeding grade boars were compared to identify the specific motion characteristics and possible structural defect in sperm morphology of the low breeding grade boar that can be easily applied as a parameter to exclude low quality sperm in AI centers.

\section{MATERIALS AND METHODS}

\section{Animal selection and preparation of semen}

Normal and low breeding grade boars were selected from Darby genetics Inc. (Anseong, Korea) on the basis of AI breeding performance. The three selected boars were Landrace pigs. As shown in Table 1, semen from boar number 2012 showed a pregnancy rate of $49.7 \%$, but semen from boar number 2004 and 2023 showed pregnancy rates greater than $70 \%$ by AI. Although delivery rates were approximately $50 \%$ for all boars, the average litter size and suckling piglets from boar 2012 were lower than those of boar 2004 and 2023 (Table 1). Based on these breeding performances, boar number 2004 and 2023 were regarded as normal and boar number 2012 as a low-breeding grade animal. In addition, motion characteristics of sperm from boar 2012 were compared to motion characteristics of sperm from boar 2004 and 2023 as positive control. Freshly ejaculated semen was immediately diluted in Beltsville thawing solution and sperm motility was measured. Spermatozoa with an initial motility of more than $75 \%$ were used in further analysis.

\section{Evaluation of sperm parameters}

A $20 \mu \mathrm{L}$ aliquot spermatozoa was placed into a prewarmed slide glass and analyzed by computer assisted semen analysis (CASA, Hamilton Thorne, Beverly, MA, USA) system. The parameters recorded for each sample were as follows: percentage of motile sperm, movement velocity such as rapid, medium, slow, and static, and movement characteristics such as progressiveness, average path velocity (VAP), straight line velocity (VSL), curvilinear velocity (VCL), sperm head elongation, and area rate.

\section{Acrosome staining assay by fluorescein isothiocyanate- Pisum sativum agglutinin}

Aliquots of boar sperm suspensions were smeared on glass slides and air-dried. Slides were fixed in $100 \%$ methanol for $10 \mathrm{~min}$ at room temperature. Thereafter, the smears of boar sperm suspensions were incubated with fluorescein isothiocyanate-Pisum sativum agglutinin (FITCPSA) (40 $\mu \mathrm{g} / \mathrm{mL}$ phosphate buffered saline [PBS], $\mathrm{pH} 7.4$, Sigma-Aldrich, St Louis, MO, USA; L-0770) solution for $30 \mathrm{~min}$ at room temperature in a dark moisture chamber. The unbound FITC-PSA solution was washed out 5 times by PBS. Finally, a mounting solution (DAKO, Carpinteria, CA, USA; S3025) was used to fix the boar sperm smears.

\section{In vitro fertilization and culture}

Semen samples from each boar were divided to make at least 10 aliquots and each aliquots was washed two times by centrifugation with Dulbecco's phosphate buffered saline (DPBS) supplemented with $0.1 \%$ bovine serum albumin at $2,000 \mathrm{~g}$ for $2 \mathrm{~min}$. After washing, the sperm pellet was resuspended in modified Tris-buffered medium (mTBM) (Abeydeera and Day, 1997), which was pre-equilibrated for $18 \mathrm{~h}$ at $39^{\circ} \mathrm{C}$ under $5 \% \mathrm{CO}_{2}$. Just before fertilization, the sperm motility from each aliquot was assessed and sperm

Table 1. Breeding performance of semen from boar number 2004, 2012, and 2013

\begin{tabular}{lcccccc}
\hline $\begin{array}{c}\text { Boar } \\
\text { number }\end{array}$ & $\begin{array}{c}\text { Age } \\
\text { (Month) }\end{array}$ & No. of sow for AI & $\begin{array}{c}\text { No. of pregnant sow } \\
\text { (Pregnant rate, } \%)\end{array}$ & $\begin{array}{c}\text { Delivery rate } \\
(\%)\end{array}$ & $\begin{array}{c}\text { Average } \\
\text { No. of farrow }\end{array}$ & $\begin{array}{c}\text { Average } \\
\text { No. of suckling piglet }\end{array}$ \\
\hline 2004 & 30 & 200 & $143(71.5)$ & 51 & 11.0 & 10.2 \\
2012 & 16 & 159 & $79(49.7)$ & 50 & 9.2 & 8.3 \\
2023 & 16 & 201 & $141(70.1)$ & 50 & 12.3 & 10.9 \\
\hline
\end{tabular}

$\mathrm{AI}$, artificial insemination. 
with more than $80 \%$ motility were used for in vitro fertilization (IVF). After 40 to $42 \mathrm{~h}$ of in vitro maturation of porcine oocytes, the cumulus cells were denuded by gentle pipetting with $0.1 \%$ hyaluronidase and washed three times in Tyrode's medium containing $0.05 \%$ (w/v) polyvinyl alcohol (Bavister et al., 1983). Oocytes with even ooplasm and visible first polar body were used for all experiments. Groups of 15 oocytes were randomly placed into $40 \mu \mathrm{L}$ droplets of mTBM in a $35 \times 10 \mathrm{~mm}$ Petri dish (Falcon; Becton Dickinson Labware, Franklin Lakes, NJ, USA) covered with pre-warmed mineral oil. After appropriate dilution, $5 \mu \mathrm{L}$ of the sperm suspension was added to the 40 $\mu \mathrm{L}$ droplet of fertilization medium (mTBM) to yield a final sperm concentration of $1 \times 10^{6} \mathrm{sperm} / \mathrm{mL}$. For IVF, the two step culture system (Gil et al., 2004) was used. The oocytes were co-incubated with sperm for $20 \mathrm{~min}$ at $38.5^{\circ} \mathrm{C}$ in a humidified atmosphere of $5 \% \mathrm{CO}_{2}$ and $95 \%$ air. After 20 min of co-incubation with the sperm, the loosely attached sperms were removed from the zonapellucida (ZP) by gentle pipetting. The oocytes were then washed three times in $\mathrm{mTBM}$ and incubated in $\mathrm{mTBM}$ without sperm for 5 to 6 $\mathrm{h}$ at $38.5^{\circ} \mathrm{C}$ in a humidified atmosphere of $5 \% \mathrm{CO}_{2}$ and $95 \%$ air. Thereafter, gametes were washed three times with embryo culture medium and cultured in $25 \mu \mathrm{L}$ micro-drops (10 gametes/drop) of porcine zygote medium (Yoshioka et al., 2002). The embryos in cultured drops were covered with pre-warmed mineral oil and incubated at $38.5^{\circ} \mathrm{C}$ under a humidified atmosphere of $5 \% \mathrm{O}_{2}, 5 \% \mathrm{CO}_{2}$, and $90 \% \mathrm{~N}_{2}$.

\section{Pronuclear staining}

Embryos were denuded by repeated pipetting in the presence of $1 \mathrm{mg} / \mathrm{mL}$ of hyaluronidase for 2 to $3 \mathrm{~min}$. The embryos were then fixed in $3.7 \%$ paraformaldehyde for 30 min at room temperature. For examination of pronuclear formation, embryos were treated with $5 \mu \mathrm{g} / \mathrm{mL}$ of Hoechst 33342 for $10 \mathrm{~min}$. After washing twice in PBS containing $0.1 \%$ (w/v) polyvinyl alcohol (PVA), the embryos were mounted and observed under ultraviolet light with an excitation wavelength of 340 to $380 \mathrm{~nm}$ and an emission wavelength of 435 to $485 \mathrm{~nm}$ using a fluorescence microscope (Nikon, Tokyo, Japan).

\section{Statistical analysis}

Semen from three different boars was collected at five different times, and each sample was divided in triplicated to make 15 sperm samples per one tested boar to analyze the motion parameters. Results were compared by one way analysis of variance, using SPSS statistical package version 15.0 for windows (IBM Corp., Armonk, NJ, USA). All data are expressed as means with standard deviation. The values were compared by paired $t$-test. The null hypothesis was rejected when the probability was $<0.05$.

\section{RESULTS}

\section{Comparison of viability and motion parameters between normal and low breeding grade semen}

The percentage of motile sperm from the three boars did not show any statistical difference. All three boars presented a sperm motility superior at $75 \%$ (Figure 1A). This level of initial motion parameters satisfies and is acceptable for AI. To identify the details of movement characteristics of the three semen samples, the distribution of rapid, medium, slow, and static sperm were calculated. Interestingly, semen from boar number 2012 presented a significantly reduced population of rapid sperm and an increased population of slow sperm compared to boar number 2004 and 2023 (Figure 1B and D). However, the proportion of medium and static sperm was not significantly different among the three semen samples (Figure 1C and E).

For a close inspection of detailed motion parameters, sperm progressiveness, VAP, VSL, and VCL from the three semen samples were analyzed. Percentage values of progressive sperm from boar 2004, 2012, and 2023 were $31.0 \pm 5.7 \%, 11.8 \pm 0.5 \%$, and $27.8 \pm 9.0 \%$, respectively, and the portion of progressive sperm from boar 2012 was significantly lower than that of boar 2004 and 2023 (Figure 2A). In addition, VAP and VSL of sperm from boar 2012 also showed a significant reduction compared to those of boar 2004 and 2023 (Figure 2B and C), although VCL did not show any significant difference (Figure 2D). Based on detailed motion parameter data, straightness and linearity index of sperm were calculated. As expected, sperm straightness and linearity from boar 2012 were significantly lower than those of boar 2004 and 2023 (Figure 3A and B). To identify the reason of low grade motion parameters in semen from boar 2012, morphological abnormal sperm parameters such as elongation rate and area of sperm head were observed. However, no significant difference among the three sperm samples was observed in sperm head morphologic index (Figure 3C and D).

\section{Assessment of oocyte penetration ability using in vitro fertilization assay}

To compare and identify the fertilization ability of sperm from boar 2012 and 2023, IVF was performed using in vitro matured pig oocytes. Sperm from boar 2012 and 2023 showed 3.4 and $75.45 \%$ of fertilization rate, respectively (Table 2). Interestingly, the number of oocytes presenting a pronucleus (PN) formation using sperm from boar 2012 was only four, one oocyte had 1PN and 3 oocytes had 2PN. However, sperm from boar 2023 fertilized 83 eggs out of 110 oocytes, and the number of PN in fertilized eggs varied from one to more than three (Table 2). Most of the spermatozoa from boar 2012 did not penetrate the $\mathrm{ZP}$ of oocytes and failed to form PN. They remained on the outer 
A.

Total motility

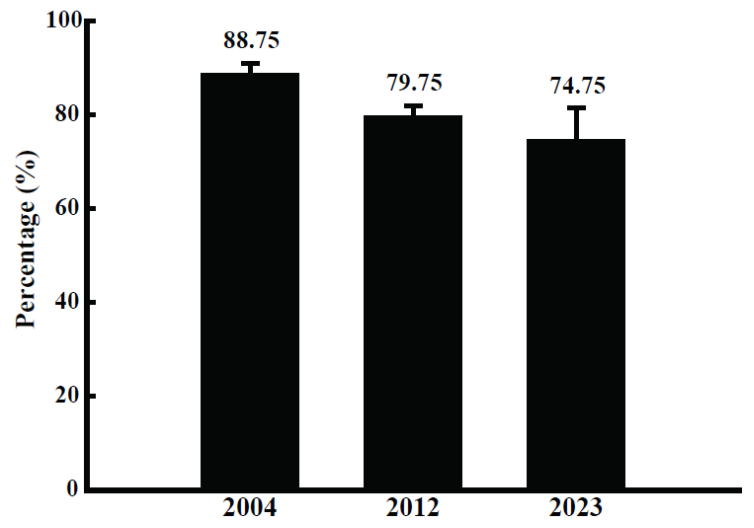

B.

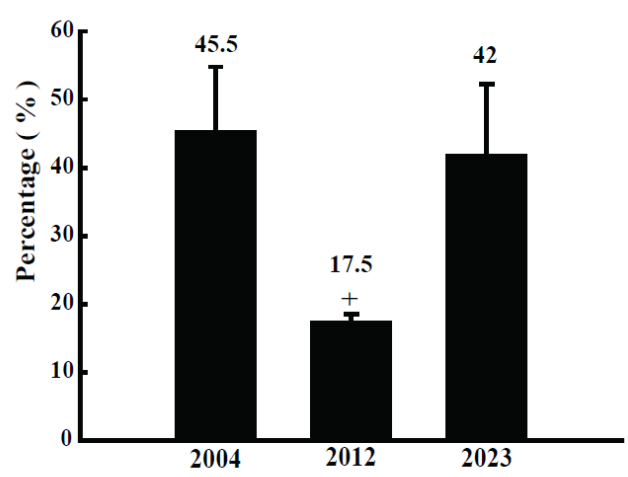

D.

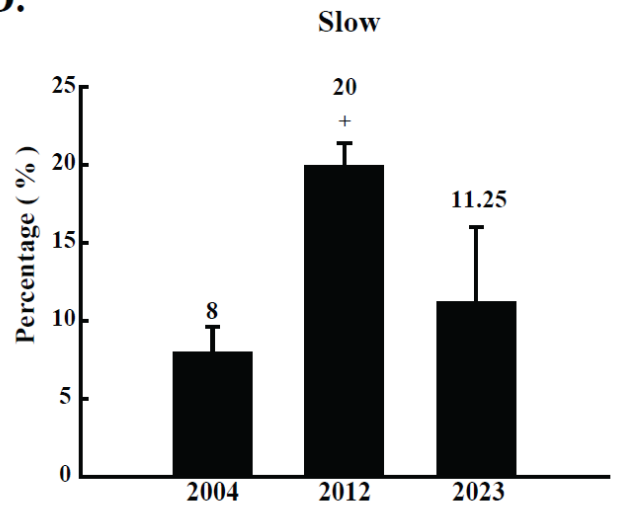

C.

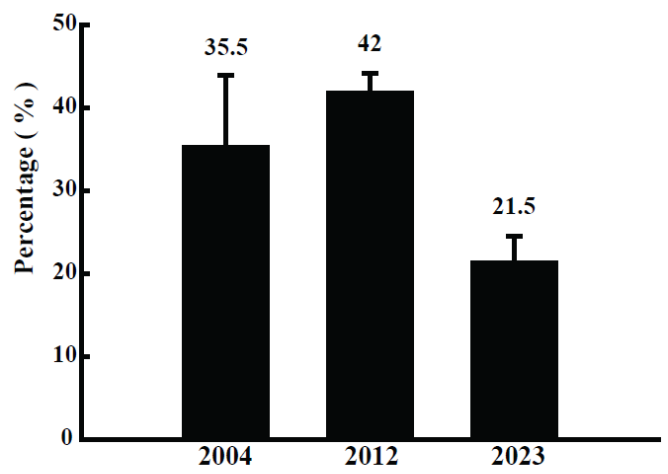

E.

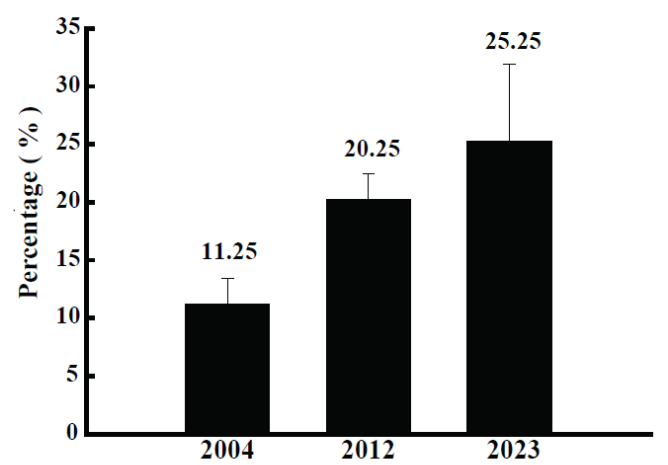

Figure 1. Percentage of motile boar sperm from boar number 2004, 2012, and 2023. (A) total motility, (B) rapidly moving sperm, (C) moderately moving sperm, (D) slow moving sperm, and (E) static sperm. Semen was collected at five different time from the boars $(\mathrm{n}=$ 5). The collected semen was divided in triplicates and applied to CASA analysis. The results are expressed as mean \pm SD. $p<0.05$. CASA, computer assisted semen analysis; SD, standard deviation.

area of the oocytes (Figure 4A). In contrast, a lower number of sperm nuclei were identified on the ZP region in fertilized oocytes with sperm from boar 2023 and PN formation was determined (Figure 4B).

\section{Comparison of acrosome formation}

To determine the reason behind the penetration defect of sperm from boar 2012, acrosome formation was analyzed using Pisum sativum agglutinin (PSA) staining. Most of the spermatozoa from boar 2023 showed a normal and full formation of the acrosome (Figure 5B). However, spermatozoa from boar 2012 showed none or broken acrosome formation (Figure 5A). Interestingly, spermatozoa from both boar 2012 and 2023 did not show severe morphological defects, such as short tail, abnormal contour, small and abnormal head, coiled or folded tail, and 
A.

Progressiveness

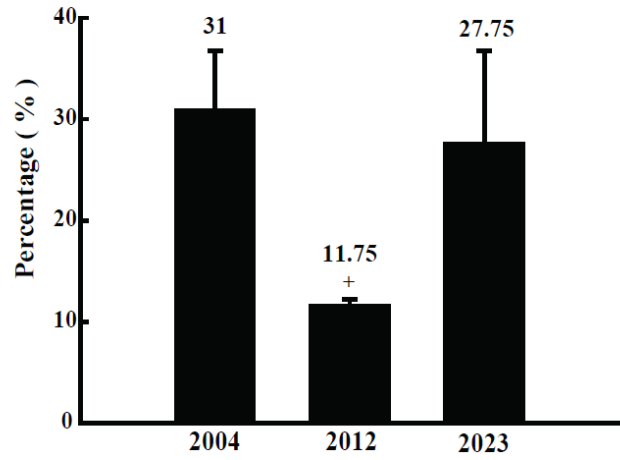

C.

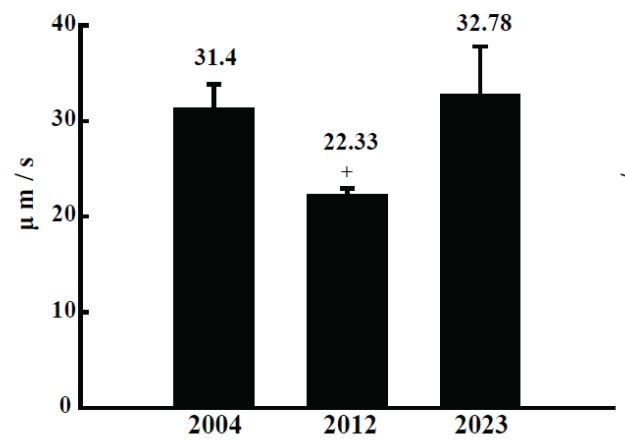

B. VAP

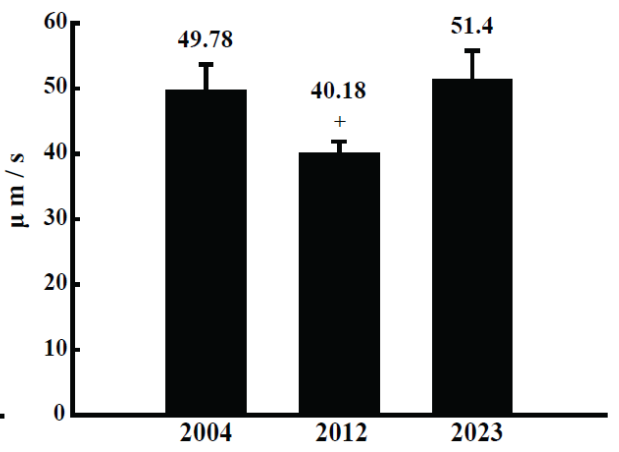

D.

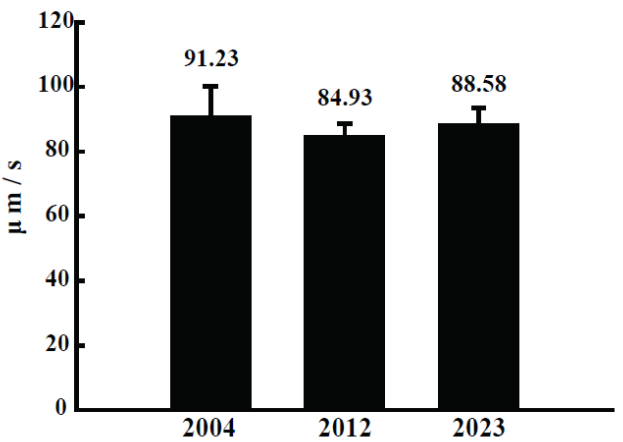

Figure 2. Comparison of detailed motion characteristics of semen from boar number 2004, 2012, and 2023. (A) progressiveness (\%), (B) average path velocity (VAP, $\mu \mathrm{m} / \mathrm{s})$, (C) straightness linear velocity $(\mathrm{VSL}, \mu \mathrm{m} / \mathrm{s})$ and (D) curve linear velocity $(\mathrm{VCL}, \mu \mathrm{m} / \mathrm{s}) \mathrm{were}$ evaluated in CASA. Semen was collected at five different time from the boars $(n=5)$. The collected semen was divided in triplicates and applied to CASA analysis. The results are expressed as mean \pm SD. $\mathrm{p}<0.05$. CASA, computer assisted semen analysis; SD, standard deviation.

underdevelopment.

\section{DISCUSSION}

The selection criteria for semen in most of the AI centers in Korea and other countries are sperm motility, concentration, and morphology (Robinson and Buhr, 2005). Sperm motility is the single most important criterion in determining fertilization rates, and normal sperm motion characteristic parameters are imperative to determine the successful fertilization of tested spermatozoa. In the present study, semen was selected based on these motility and viability parameters and AI was performed. However, the semen from boar 2012 showed very low breeding performances, as shown in Table 1. This result strongly suggests that initial parameters such as sperm motility and concentration are not reliable criteria for successful fertilization, farrowing rate, and litter size through AI. Similarly in rabbit, sperm motility and morphology based parameters showed up to $45 \%$ variability in fertility results (Lavara et al., 2005), while a combination of parameters, including sperm motility, concentration, and viability showed $16 \%$ of variability in litter size (Quintero-Moreno et al., 2007). These previous reports also suggest that more reliable qualitative parameters are required to select sperm for AI.

Although the total motility and viability did not show any statistical significance among the tested sperm, significant differences were observed in percentage of rapid and slow moving sperm, indicating that semen from boar 2012 had a reduced number of rapid moving spermatozoa and an increased number of slow moving spermatozoa (Figure 1B and D, respectively). The percentage of motile sperm and sperm velocity has been well correlated to reproductive success. Thus, assessing both spermatozoa velocity of movement and total motility is recommended for the selection of qualitative semen for AI. In addition to sperm motility, CASA can automatically analyze other sperm motion characteristic parameters such as VCL, VAP, VSL, linearity (LIN), and straightness (STR). Although VCL did not show any significant difference among the tested semen samples, semen from boar 2012 showed a significant decreased in progressive sperm number, VAP, and VSL compared to semen from boar 2004 and 2023 
A.

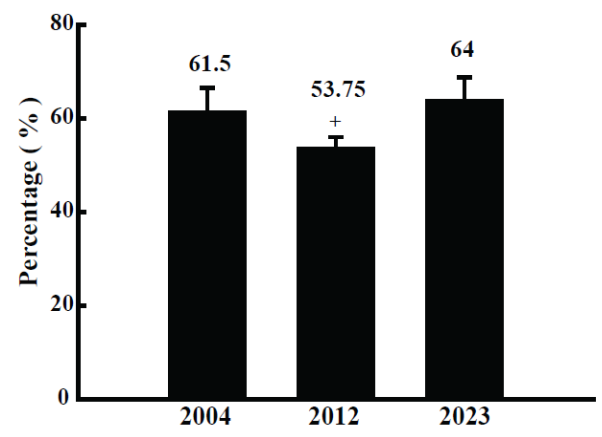

C.

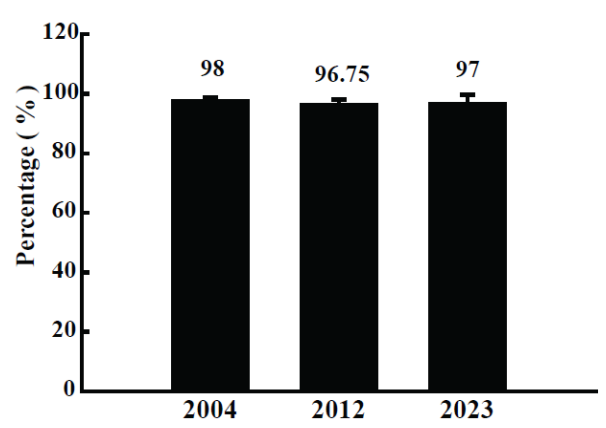

B.

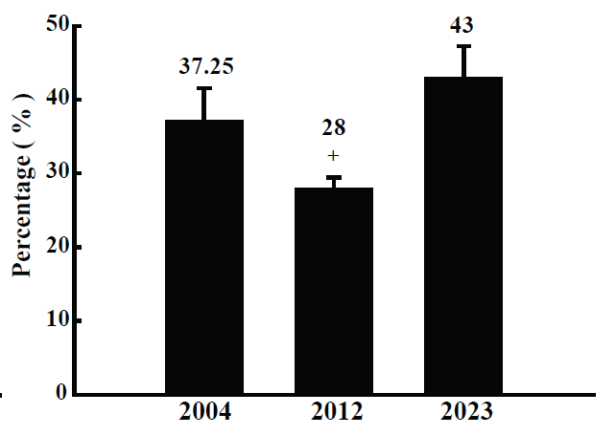

D.

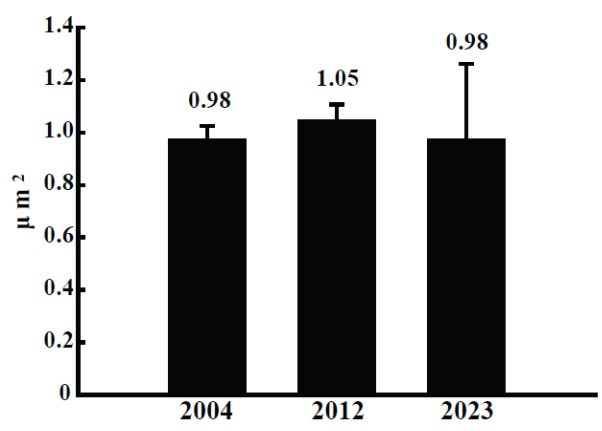

Figure 3. Comparison of motion and morphological index. Two motion indices were evaluated for boar 2004, 2012, and 2023. (A) straightness (STR, \%) was calculated as the ratio of straight-line velocity (VSL) to average path velocity (VAP), and (B) linearity (LIN, \%) was defined as the ratio of VSL to curvilinear velocity (VCL). Morphological abnormality was evaluated by measuring (C) sperm head elongation ratio (\%) and (D) area $\left(\mu \mathrm{m}^{2}\right)$. Elongation was calculated by the ratio of minor to major axis of sperm head, and area corresponded to the head size. Semen was collected at five different times from the boars $(n=5)$. The collected semen was divided in triplicates and applied to CASA analysis. The results are expressed as mean \pm SD. $p<0.05$. CASA, computer assisted semen analysis; $\mathrm{SD}$, standard deviation.

(Figure 2). These data also represent a possible correlation between breeding performance and sperm motion characteristics, and sperm presenting a low progressive rate as well as reduced VAP and VSL compared to the other semen samples should not be considered for AI. Among the motion parameters, progressive forward motility presents a positive relationship with boar fertility (Ivanova and Mollova, 1993; Flowers, 1997). Consequently, it is noticeable that not only the sperm motility and concentration but also detailed motion parameters such as VAP, VCL, and VSL should be considered to select qualitative semen for field AI to enhance boar breeding performance.

However, STR can be variable (increase or decrease) depending on the sperm swimming pattern because it is defined as the ratio of VSL to VAP. Similarly, LIN is defined as the ratio of VSL to VCL. Therefore, these parameters are probably not very useful for detecting abnormal sperm motion because of their low sensitivity and the fact that they are calculated from other derived parameters (Ha et al., 2013).

Sperm abnormality has been related to male infertility and sterility in most species, and abnormalities associated with acrosome defects were reported in human, bulls, boars, stallions, rams, and dogs (Hurtgen and Johnson, 1982; Aitken et al., 1990; Toyama and Itoh, 1993; Soderquist, 1998; Chenoweth, 2005; Moretti et al., 2005; Santos et al., 2006). It has been reported that abnormal sperm may reduce fertility because of i) the failure to reach the fertilization site, or ii) the inability to fertilize the ovum or to sustain

Table 2. Analysis of sperm fertilization ability using IVF

\begin{tabular}{lccccccc}
\hline Type of sperm & No. of oocytes & \multicolumn{3}{c}{ No. of oocytes with } & \multicolumn{2}{c}{ of fertilized } \\
\cline { 3 - 7 } (Boar number) & for IVF & 1 PN & 2 PN & 3 PN & >3PN & PN formation & oocytes \\
\hline Normal (2023) & 110 & 5 & 25 & 26 & 28 & 83 & 75.45 \\
Defect (2012) & 119 & 1 & 3 & 0 & 0 & 4 & 3.4 \\
\hline
\end{tabular}

IVF, in vitro fertilization; PN, pronucleus. 
A.
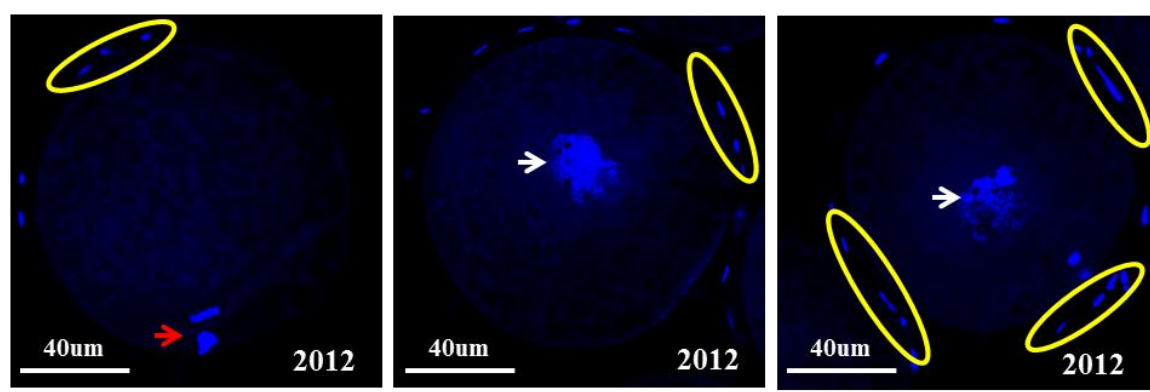

B.
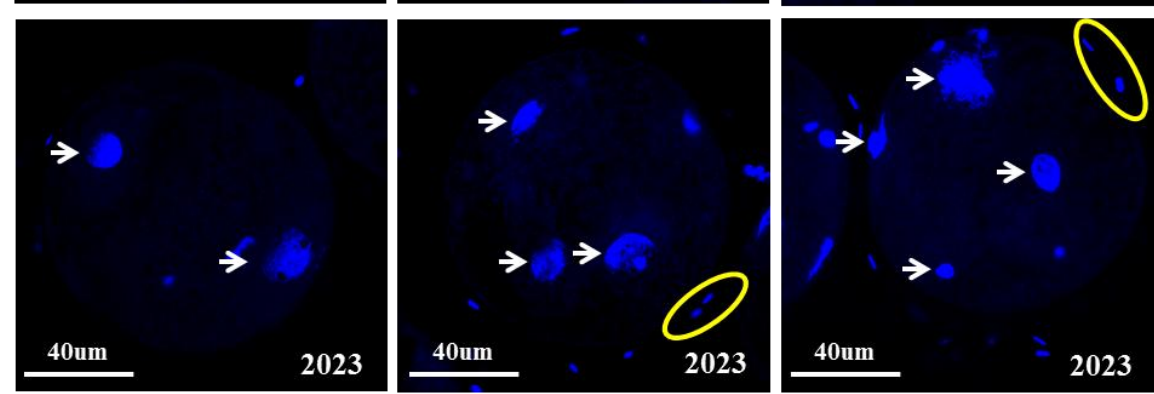

Figure 4. Comparison of pronucleus formation after in vitro fertilization of porcine oocytes. (A) Porcine oocytes in vitro fertilized with sperm from boar number 2012 and (B) 2023. White arrows indicate pronuclei, the red arrow indicates a polar body of unfertilized oocytes, and yellow circles indicate non-fertilized sperm heads containing nucleic acid (4',6-diamidino-2-phenylindole, $\times 100)$.

A.
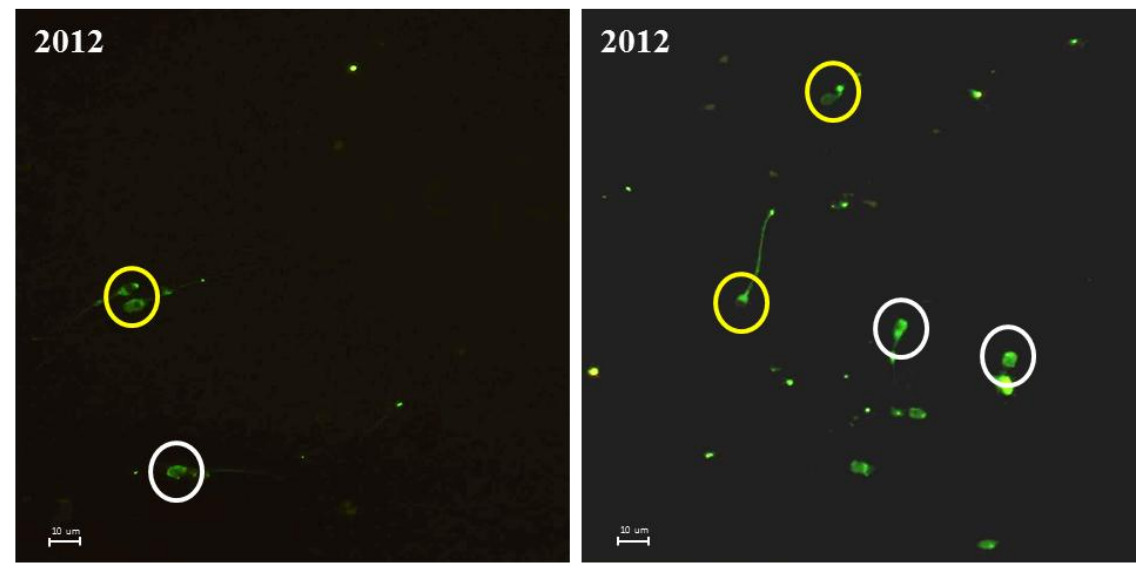

B.
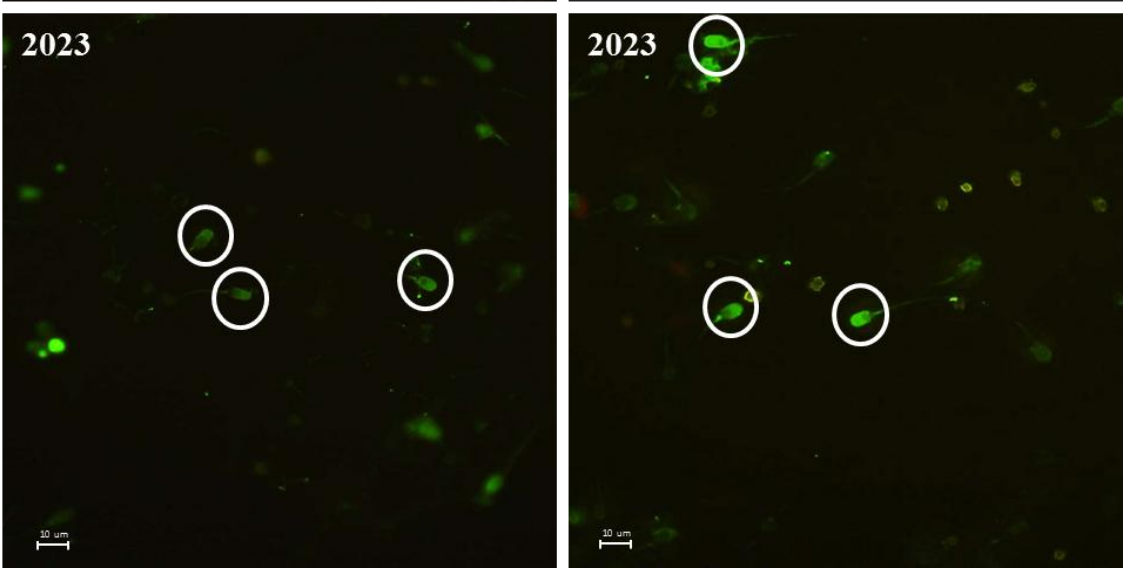

Figure 5. Comparison of acrosome formation in sperm from boar number 2012 and 2023. Acrosome was stained with FITC-PSA probe. (A) sperm from boar 2012, and (B) sperm from boar 2023. Yellow circles show the spermatozoa with acrosome defect and white circles contain the sperm head formed with normal acrosome (FITC-PSA, ×400). FITC-PSA, Fluorescein isothiocyanate-Pisum sativum agglutinin . 
development of the early embryo (Saacke, 1990). In the first case, the matter of failure to reach the fertilization site is closely related to the motion parameter characteristics, and this kind of defects can be solved by increasing the number of sperm used in AI (Saacke, 1990). However, the second case cannot be solved by an increase in sperm numbers, since it is caused by other problems such as genetic defects (Chenoweth, 2005).

In the present study, fertilization ability of boar 2012 using IVF assay was very low compared to boar 2023. Pronucleus formation of sperm from boar 2012 showed only $3.4 \%$ (Table 2), indicating that most of the sperm from boar 2012 had a critical penetration defect into the oocyte ZP. In general, when spermatozoa reach the oocyte, they initiate the acrosome reaction and release an enzyme called acrosin, which degrades the oocyte $\mathrm{ZP}$ to allow spermatozoa to penetrate into the oocyte cytosol, where the fertilization is finally completed. However, most of the sperm from boar 2012 did not penetrate the oocytes (Figure 4A), suggesting that spermatozoa from boar 2012 present a certain acrosome defect. Consequently, acrosome staining data using PSA staining method revealed that sperm from boar 2012 showed an abnormal formation of the acrosome compared to boar 2023. These data strongly indicated that the acrosome defect in sperm from boar 2012 caused poor motion characteristics and IVF failure.

The causes of acrosome defect may be environmental, genetic, or a combination of both (Chenoweth, 2005), and it is difficult to eradicate structurally defective spermatozoa from the semen before $\mathrm{AI}$ at any rate. However, it is essential to increase the probability to select normal and high quality semen by simple and easy observations that can be performed at the AI center. In this regard, the present study recommends two simple and easy methods to select normal grade sperm together with primary parameters such as viability and concentration. These methods are the observation of detailed motion parameters and acrosome staining. Most of the AI centers have a CASA assay system, and CASA can provide not only basic information such as motility, viability, and concentration, but also valuable detailed motion parameters such as progressiveness, VAP, and VSL. Based on this information, low quality semen may be discriminated using CASA analysis. Then, semen containing spermatozoa with an acrosome defect can be eliminated by simple PSA staining which takes only $30 \mathrm{~min}$, at most.

In summary, we compared the semen from boars presenting low and normal breeding performance. Although the low breeding performance semen presented similar viability and motility compared to the normal breeding performance semen, its detailed motion characteristics, such as progressiveness, VAP, and VSL were lower. In addition, acrosome staining using PSA assay clearly determined the defect of acrosome formation in the semen showing low breeding performances. Thus, the present study strongly recommend the inclusion of detailed motion parameter analysis and acrosome staining before selection of semen for AI to enhance the semen quality and breeding performance.

\section{ACKNOWLEDGMENTS}

This study was supported by grant PJ009535 from the Next-Generation BioGreen 21 program, Rural Development Administration, Republic of Korea.

\section{REFERENCES}

Abeydeera, L. R. and B. N. Day. 1997. In vitro penetration of pig oocytes in a modified Tris-buffered medium: Effect of BSA, caffeine and calcium. Theriogenology 48:537-544.

Aitken, R. J., L. Kerr, V. Bolton, and T. Hargreave. 1990. Analysis of sperm function in globozoospermia: implications for the mechanism of sperm-zona interaction. Fertil. Steril. 54:701707.

Amann, R. P. 1989. Can the fertility potential of a seminal sample be predicted accurately? J. Androl. 10:89-98.

Bavister, B. D., M. L. Leibfried, and G. Lieberman. 1983. Development of preimplantation embryos of the golden hamster in a defined culture medium. Biol. Reprod. 28:235247.

Blasco, L. 1984. Clinical tests of sperm fertilizing ability. Fertil. Steril. 41:177-192.

Bostofte, E., J. Serup, and H. Rebbe. 1984. Interrelations among the characteristics of human semen, and a new system for classification of male infertility. Fertil. Steril. 41:95-102.

Chenoweth P. J. 2005. Genetic sperm defects. Theriogenology 64:457-468.

Correa, J. R. and P. M. Zavos. 1994. The hypoosmotic swelling test: Its employment as an assay to evaluate the functional integrity of the frozen-thawed bovine sperm membrane. Theriogenology 42:351-360.

Flowers, W. L. 1997. Management of boars for efficient semen production. J. Reprod. Fertil. Suppl. 52:67-78.

Foldesy, R. G., M. M. Vanderhoof, and D. W. Hahn. 1986. Lack of correlation between fertility and sperm numbers in male rats treated with histrelin, a potent LHRH agonist. J. Androl. 7:140-146.

Gil, M. A., M. Ruiz, J. M. Vazquez, J. Roca, B. N. Day, and E. A. Martinez. 2004. Effect of short periods of sperm-oocyte coincubation during in vitro fertilization on embryo development in pigs. Theriogenology 62:544-552.

Glass, R. H. and R. J. Ericsson. 1979. Spontaneous cure of male infertility. Fertil. Steril. 31:305-308.

Hoing, L. M., P. Devroey, and A. C. Van Steirteghem. 1986. Treatment of infertility because of oligoasthenoteratospermia by transcervical intrauterine insemination of motile spermatozoa. Fertil. Steril. 45:388-391.

Ha, W. T., W. Y. Lee, R. Lee, J. H. Kim, N. H. Kim, J. H. Kim, I. J. Lee, and H. Song. 2013. Effect of naturally derived substances 
on motion parameters of in vitro non-freezing preserved pig sperm. Reprod. Dev. Biol. 37:9-16.

Hurtgen, J. P. and L. A. Johnson. 1982. Fertility of stallions with abnormalities of the sperm acrosome. J. Reprod. Fertil. Suppl. 32:15-20.

Ivanova, M. and M. Mollova. 1993. Zona-penetration in vitro test for evaluating boar sperm fertility. Theriogenology 40:397-410.

Lavara, R., E. Moce, F. Lavara, M. P. Viudes de Castro, and J. S. Vicente. 2005. Do parameters of seminal quality correlate with the results of on-farm inseminations in rabbits? Theriogenology 64:1130-1141.

Moretti, E., G. Collodel, G. Scapigliati, I. Cosci, B. Sartini, and B. Baccetti. 2005. 'Round head' sperm defect. Ultrastructural and meiotic segregation study. J. Submicrosc. Cytol. Pathol. 37:297-303

Nicander, L. and A. Bane. 1966. Fine structure of the sperm head in some mammals, with particular reference to the acrosome and the subacrosomal substance. Z. Zellforsch. Mikrosk. Anat. 72:496-515.

Quintero-Moreno, A., T. Rigau, and J. E. Rodriguez-Gil. 2007. Multivariate cluster analysis regression procedures as tools to identify motile sperm subpopulations in rabbit semen and to predict semen fertility and litter size. Reprod. Domest. Anim. 42:312-319.
Robinson, J. A. and M. M. Buhr. 2005. Impact of genetic selection on management of boar replacement. Theriogenology 63:668678

Saacke, R. G. 1983. Semen quality in relation to semen preservation. J. Dairy Sci. 66:2635-2644.

Saacke, R. G. 1990. What is abnormal? And is abnormal dependent upon the animal. In: Proceeding of the NAAB 13th Technical Conference on AI and Reprod. Milwaukee, WI, USA pp. 67-73.

Santos, N. R., N. Krekeler, A. Schramme-Jossen, and D. H. Volkmann. 2006. The knobbed acrosome defect in four closely related dogs. Theriogenology 66:1626-1628.

Soderquist, L. 1998. Reduced fertility after artificial insemination in a ram with a high incidence of knobbed acrosomes. Vet. Rec. 143:227-228.

Toyama, Y. and Y. Itoh. 1993. Ultrastructural features and pathogenesis of knobbed spermatozoa in a boar. Am. J. Vet. Res. 54:743-749.

World Health Organization. 1992. WHO Labboratory manual for the Examination of human semen and semen-cervical mucus interaction. Cambridge University Press, New York, NY, USA.

Yoshioka K., C. Suzuki, A. Tanaka, I. M. Anas, and S. Iwamura. 2002. Birth of piglets derived from porcine zygotes cultured in a chemically defined medium. Biol. Reprod. 66:112-119. 